\title{
Characterization of Maritime Pine Forests with Combination of Simulated P-Band SAR Data and Hyperspectral Data
}

\author{
C. Albinet, ${ }^{1}$ P. Borderies, ${ }^{1}$ and J. P. Gastellu-Etchegorry ${ }^{2}$ \\ ${ }^{1}$ Electromagnetism and Radar Department, Radio-Communication and Propagation research Unit, Office National d'Études et de \\ Recherches Aérospatiales (ONERA), 2 avenue Edouard Belin, BP7 4025, 31055 Toulouse Cedex 4, France \\ ${ }^{2}$ Centre d'Etudes Spatiales de la Biosphère (CESBIO), 18 avenue Edouard Belin, BP 2801, 31401 Toulouse Cedex 9, France
}

Correspondence should be addressed to C. Albinet, clement.albinet@onera.fr

Received 22 June 2012; Accepted 21 October 2012

Academic Editor: Hosam El-Ocla

Copyright () 2012 C. Albinet et al. This is an open access article distributed under the Creative Commons Attribution License, which permits unrestricted use, distribution, and reproduction in any medium, provided the original work is properly cited.

\begin{abstract}
This paper describes a sensitivity study performed on simulated radar and optical remote sensing forest data. It presents how the dual model has been built up. The first step is a forest growth model fed with biophysical parameters. The geometrical description is then the input of an optical hyperspectral model, giving reflectance spectra, and a Synthetic Aperture Radar (SAR) model, giving the polarimetric and interferometric observables. As an illustration, the first results obtained by both models outputs are presented, and fusions of these outputs are performed.
\end{abstract}

\section{Introduction}

The retrieval of biophysical parameters of forests with remote sensing is nowadays a challenge. In particular, forest biomass and soil and branches moisture content are three parameters of interest. It is well known that low frequency radars may provide lots of characteristics of forests, and in particular P-band is often proposed for biomass estimation [1]. On the other hand, spectral signatures provided by optical measurements are able to deliver features of forest vegetation, like Leaf Area Index (LAI) [2], to derive optical indexes like the Normalized Difference Vegetation Index (NDVI), and can be used to determinate tree species [3].

Hopefully, using both sources of information through a combination process should improve the determination of the characteristic parameters of forest [4]. To evaluate the potential of this combined approach, a focus is done in this paper on a parallel direct modeling approach in which the same forest scenario is simulated in polarimetric P-band backscattering and in optical bands. This parallel modeling approach was described and validated in a previous paper [5], and here the focus will be made on its results.

In Section 2 the general functioning of the parallel modeling, with the inputs and links between the models, is described. Then, in Section 3 a sensitivity study is carried out with the radar model only on a forest with different biomass, soil moisture content, and branches moisture content. Section 4 consists of a sensitivity study with the optical model. Finally, the results carried out on previous radar and optical data, of a nonsupervised and of a supervised data analyses, are, respectively, shown in Sections 5 and 6.

\section{Parallel Modeling}

2.1. Model Organization. The radar and optical modeling is considered as parallel because the geometrical 3D description of the forest is the same for both models. In fact, a ground representation of a pine forest as a function of growing age and consequently growing biomass is obtained thanks to a growth model depicted in [6], which delivers LAI and the statistical parameters of the trunks and branches in terms of size, location, and orientation, for a given biomass [7].

It is necessary to clarify that the biomass considered in this paper is the sum of branches and trunks biomass, as leaves biomass is considered as negligible.

As shown in Figure 1, this geometrical information is then used for MIPERS (Multistatic Interferometric Polarimetric Electromagnetic Model for Remote Sensing) 


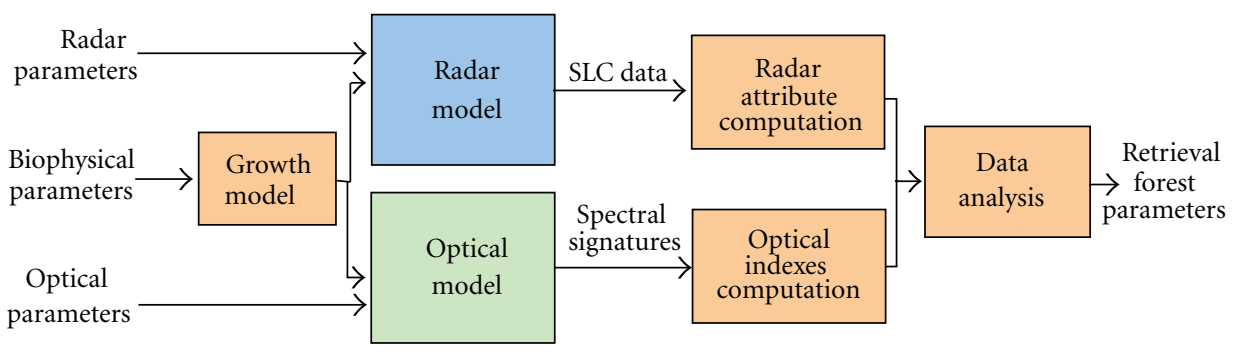

FIGURE 1: General diagram of the parallel radar and optical models.

simulations of the radar backscattering matrix [8] and for DART (Discrete Anisotropic Radiative Transfer) simulations of the scene reflectance and 3D radiative budget [9].

\subsection{Model Adaptation. The two models have different ways of functioning.}

For the radar model MIPERS, the scatterers are represented by finite length cylinders for the trunks and branches, as shown in Figure 2.

At P-band, the wavelength is $75 \mathrm{~cm}$ so the leaves will not have any influence and so have not been considered in the simulation.

For the optical model DART, the trees and the soil are represented by two types of elements: volume (for leaves) and surface (for trunks, branches, and ground) elements, as shown in Figure 3 for the representation of buildings.

In order to have radar and optical scene as close as possible, the cylinders of the radar model are replaced by $3 \mathrm{D}$ elements made of polygons.

A sensitivity study on scene reflectance was performed with $3 \mathrm{D}$ representations of cylinders with 8 to 24 polygons per cylinder and showed that 8 polygons per cylinder were enough to correctly represent the whole scene made of approximately 100.000 cylinders [5]. Leaves are represented by volumes with an LAI given by the growth model. The optical model uses a discrete ordinate ray tracing method for simulating the scene bottom of atmosphere (BOA) hyperspectral reflectance.

\subsection{Radar and Optical Simulation Ground Parameters. Radar} simulations have been carried out on an 80 by $80 \mathrm{~m}$ wide scene, with a resolution of $1 \mathrm{~m}$, at P-band $(430 \mathrm{MHz})$. Branches and trunks are represented by homogeneous cylinders, and the soil is considered as flat. Moisture contents (m.c.) for the soil, branches, and trunks are inputs of the model.

For each pixel of the Single Look Complex (SLC) data simulated, the coherent sum of all the scatterers response is computed, taking into account direct contribution from soil and double bounce, all of them with the proper attenuation. An average value on the whole scene is then obtained for all polarizations.

For optical simulations, the model has been adapted to be able to simulate forest the same way as the radar model. Branches and trunks are represented by $3 \mathrm{D}$ objects made of surfaced polygons, and leaves are represented by

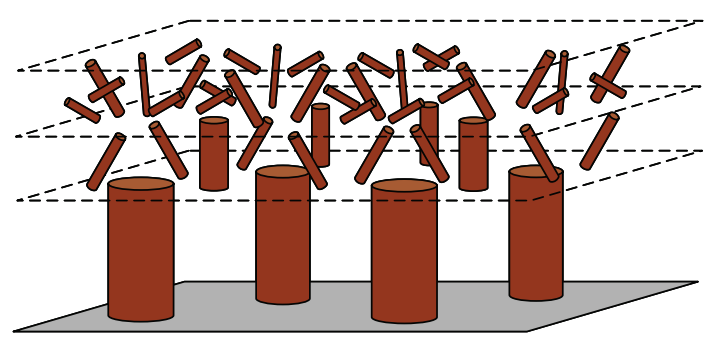

FIGURE 2: Scatterers representation in the radar model.

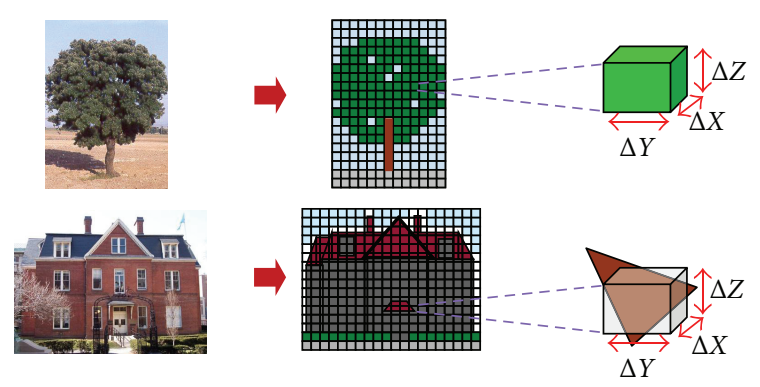

FIGURE 3: Representation of volume (up) and surfaces (down) in the optical model.

homogenous 3D volumes. The leaves moisture content in optical simulations is linked to the branches moisture content in radar simulations. For soils, the moisture content is the same for both kinds of simulations.

Optical simulations use the following parameters.

(i) Leaves of the undergrowth are made of spectral signature provided by the ASTER database [10].

(ii) PROSPECT [11] model is coupled with DART to simulate the impact of tree leaves moisture content on scene spectral signature.

(iii) Spectral signature for trunks and branches was measured on pine bark in 2002 [12].

(iv) The ground is made of bare soil. The following hypothesis is done: a bare soil corresponds to a ground with a null biomass ( 0 ton/ha). The spectral information is extracted from database developed at the ONERA and including spectral signature measured in laboratory of bare soils with several moisture content [13]. 
(v) The scene is $12 \mathrm{~m}$ by $12 \mathrm{~m}$ wide, which is sufficient, because the scene is homogeneous and there is no speckle effect in optical data. Simulations are performed, with a spatial resolution of $4 \mathrm{~m}$, for 160 spectral bands over the entire reflective spectral domain $(0.4-2.5 \mu \mathrm{m})$. These parameters are common with those considered for airborne campaigns at the ONERA [14].

Table 1 shows the values of biophysical parameters considered as input of radar and optical models.

Note that in order to simplify the simulations we assume that leaves m.c. and branches m.c. are evolving together, as shown in Table 1, as optical data tolerate a delay between acquisitions [15].

For every pixel, and every spectral band, a bottom of atmosphere reflectance is computed by DART. The average values of reflectance for each band are then combined to obtain the spectral signature.

With these spectral signatures, three optical indexes are computed:

(i) the Normalized Difference Vegetation Index (NDVI), linked to the LAI [16];

(ii) the Global Vegetation Moisture Index (GVMI), linked to the leaves moisture content [17];

(iii) the NINSOL, an optical index developed at the ONERA [18] and sensitive to the soil moisture content.

\section{Radar Simulations}

Figure 4 represents the evolution of polarimetric backscattering as a function of biomass for various soil moisture contents, and Figure 5 represents the evolution of polarimetric backscattering as a function of biomass and branches moisture content.

Soil moisture content varies between $0 \%$ for very dry soils and more than $39 \%$ for very wet, saturated soils, with average values for forests around 20\%. In Figure 4, the average values of biomass are kept for vegetation, and only soil moisture content is parameterized. Actually, soil moisture content is supposed to vary independently of vegetation moisture content. It can be seen that this parameter poorly influences $\mathrm{HV}$, has a strong influence on VV for low ages and a reduced one at higher ages, and is overall prominent for $\mathrm{HH}$.

Actually, $\mathrm{HH}$ intensity follows the same evolution with different offsets. For usual values of moisture content, around $25 \%$, the impact is low. We can notice that HV intensity is poorly influenced by soil characteristics, which is expected.

For branch moisture content, according to the literature [19], it looks reasonable to consider a variation between a ceiling value of $40 \%$ and a top value of $70 \%$.

In this case, Figure 5 shows a very strong influence of this parameter on the polarimetric response in P-band for all polarizations. Actually, as branch moisture content increases, VV and HV increase but also canopy extinction increases
TABLE 1: Biophysical parameters for the simulations.

\begin{tabular}{lcccccc}
\hline Biophysical parameters & \multicolumn{7}{c}{ Simulations } \\
\hline Age of the forest (yr) & 6 & 16 & 26 & 36 & 46 & 56 \\
Total biomass (ton/ha) & 14 & 50 & 88 & 114 & 128 & 133 \\
LAI $\left(\mathrm{m}^{2} / \mathrm{m}^{2}\right)$ & 5.4 & 5.1 & 4.4 & 3.3 & 2.9 & 2.5 \\
Leaves m.c. (\%) & 39 & 49 & 60 & 71 & 81 & 92 \\
Branches m.c. (\%) & 40 & 46 & 52 & 58 & 64 & 70 \\
Volume m.c. index & 1 & 2 & 3 & 4 & 5 & 6 \\
Soil m.c. (\%) & 0 & 18 & 24 & 29 & 34 & 39 \\
\hline
\end{tabular}

Age of the forest, total biomass, and LAI are dependent to each other. Leaves m.c. and branches m.c. are also dependent on each other. On the opposite, soil m.c. is independent of the other parameters.

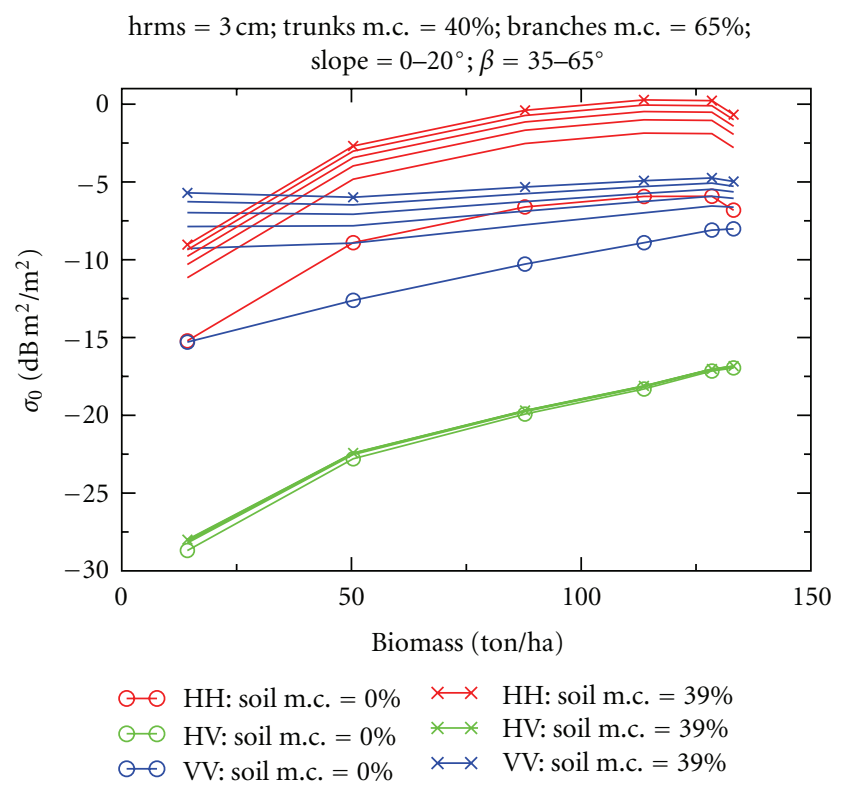

FIGURE 4: Radar polarimetric radiometry for several biomasses and soil moisture content, as given in Table 1.

which makes the trunk double bounce with soil decrease which mainly corresponds to $\mathrm{HH}$.

It can be seen that the influence of the input parameters on the output ones is complex and that incorporating in the analysis additional data may be fruitful.

\section{Optical Simulations}

4.1. Optical Simulations for Forest without Leaves. First the scene is composed of only flat soil, trunks, and branches. No leaves are considered, which corresponds to winter season for deciduous trees.

Figure 6 shows the evolution of the spectral reflectance, on such a temperate forest with a ground cover (grass rye), for several biomasses corresponding to growing ages.

The soil spectrum and the bark one are plotted also. One can see that the shape of the various scene spectra follows the soil one all the more so as the biomass is low. An explanation of this result is that as forest age is growing, the number of branches per unit area decreases, in spite of an increase 


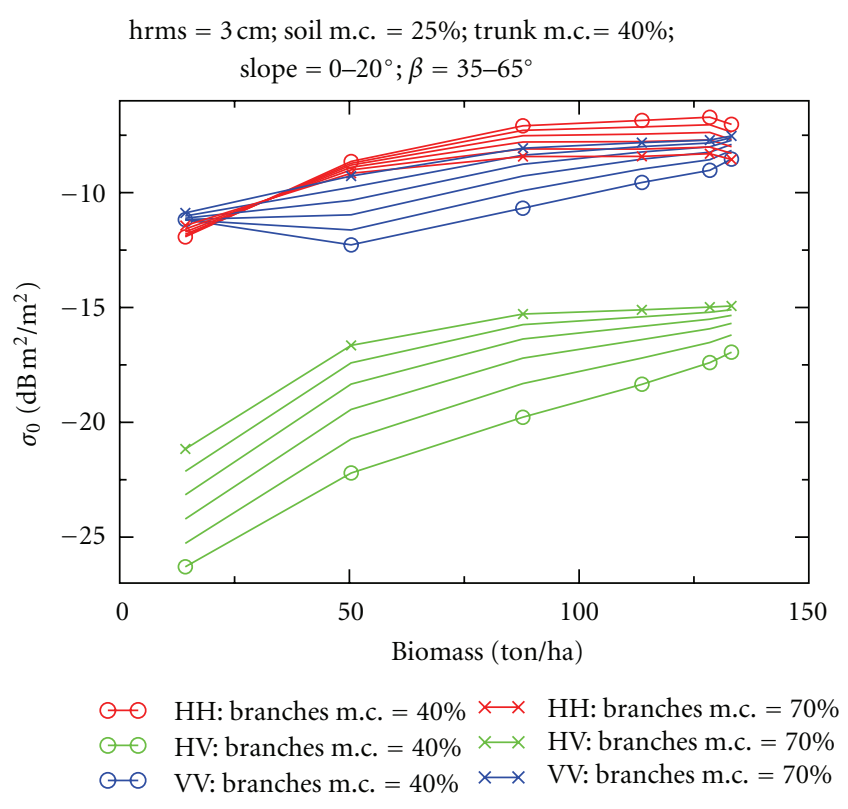

FIGURE 5: Radar polarimetric radiometry for several biomasses and branches moisture content.

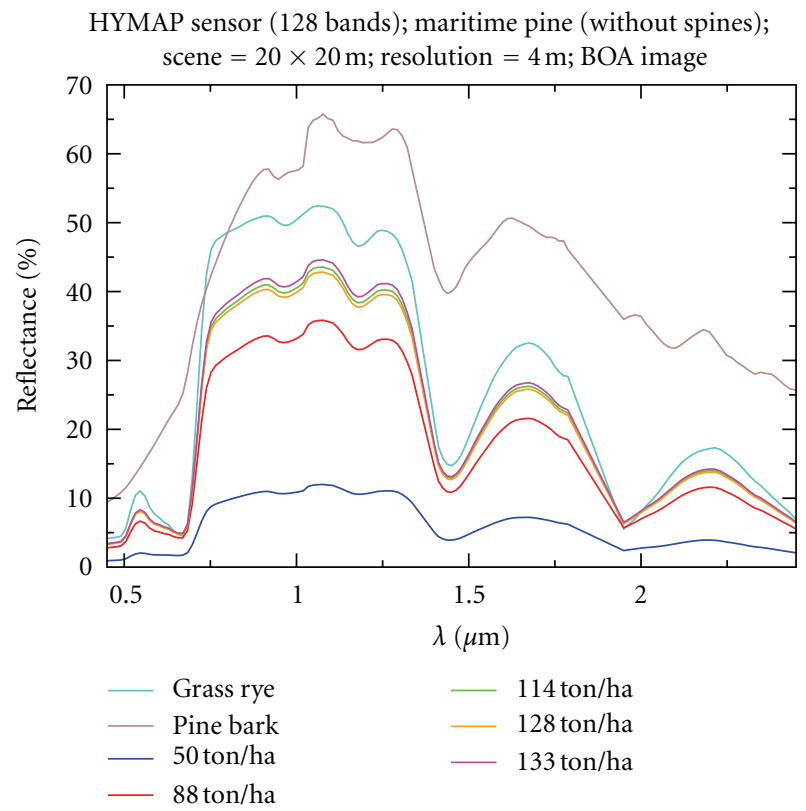

Figure 6: Reflectance spectra of the whole scene for different biomasses, as well as for the bare organic soil and for the bark.

in branches weight, and then the part of soil in the images decreases.

It is coherent with the fact that barks spectrum presents low influence, except for small biomasses.

Figure 7 deals with the same forests with a mineral soil with two moisture contents: a dry one (m.c. ideally considered at $0 \%$ ) and a wet one (m.c. of $30 \%$ ). One can see that the spectral reflectance of the whole scene tightly follows the soil spectra.

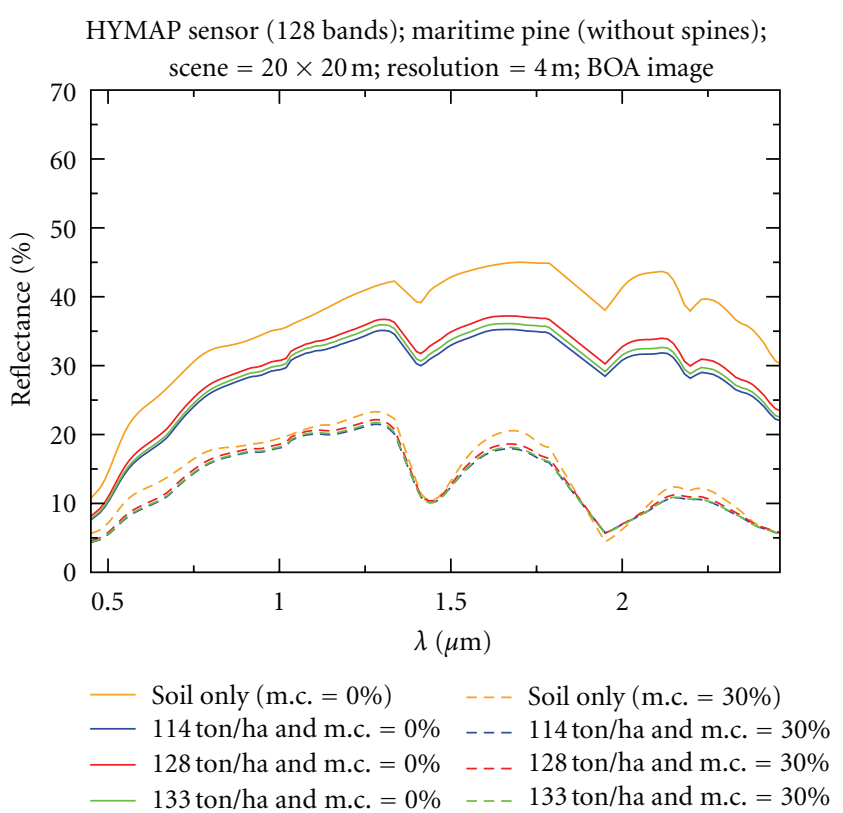

Figure 7: Reflectance spectra simulated for several ages of forest and a mineral soil with a $0 \%$ m.c. for the first one and a $39 \%$ m.c. for the other one. In addition, the measured reflectance spectra of the soil for both m.c. are displayed in orange.

Comparing both scenes, it is clear that scene reflectance spectra are more sensitive to biomass for organic soil than for mineral ones, which can be explained by the fact that the pine bark spectrum is closer to the mineral soil spectrum than to the grass rye one.

We can see here that the spectral reflectance of the scene is more affected by the change in soil moisture content than in biomass.

4.2. Optical Simulations for Forest with Needles. Here leaves, needles as a matter of fact, are considered. For the first simulation, several soil spectral signatures of the same kind of soil with moisture content from $0 \%$ for a dry soil to $39 \%$ for a wet soil, have been chosen as the soil spectral signature. The biomasses are identical to those used for radar simulations. The optical indexes have been computed for the previous parameters and are shown in Figure 8.

One can notice that the NDVI is a little bit sensitive to the biomass evolution and independent of the soil moisture content parameter. In fact, when the biomass is increasing, the LAI is decreasing, because as each tree biomass is growing, their density is decreasing, so the part of the soil in the pixel is more and more important.

For same reason, the NINSOL is more sensitive to soil moisture content for high biomass.

For the second simulation, several leaves moisture contents have been simulated with PROSPECT, from 39\% to $92 \%$, and the corresponding optical signatures have been chosen as the leaves spectral signature. The biomass varies the same way as previously. The result is shown in Figure 9. 


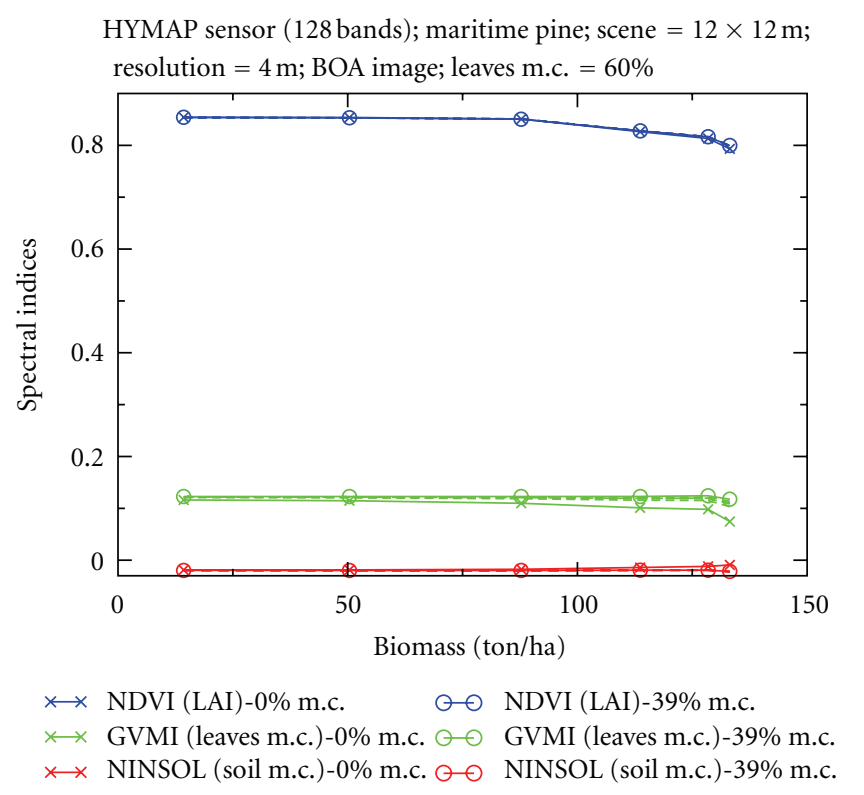

FIgURE 8: Sensitivity of spectral indices for several biomass and soil moisture content, as given in Table 1.

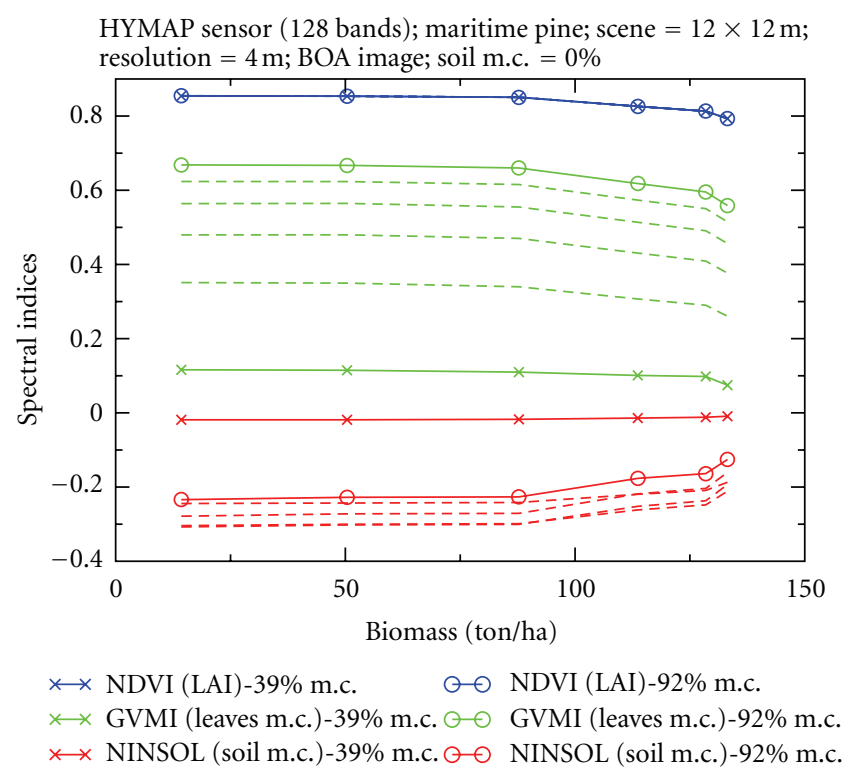

FIGURE 9: Sensitivity of spectral indices for several biomasses and leaves moisture content.

One can observe that the NDVI is not sensitive to leaves moisture content and evolutes the same way as in Figure 8.

For the GVMI, strong variations can be seen in the figure. The GVMI is much more significantly linked to leaves moisture content, than to biomass.

The NINSOL presents the same evolution with biomass and leaves moisture content. This optical index is more sensitive to leaves moisture content that it is supposed to be. An explanation is than this optical index has been designed for bare soils, which is not the case here since part of its variation is due to vegetation.

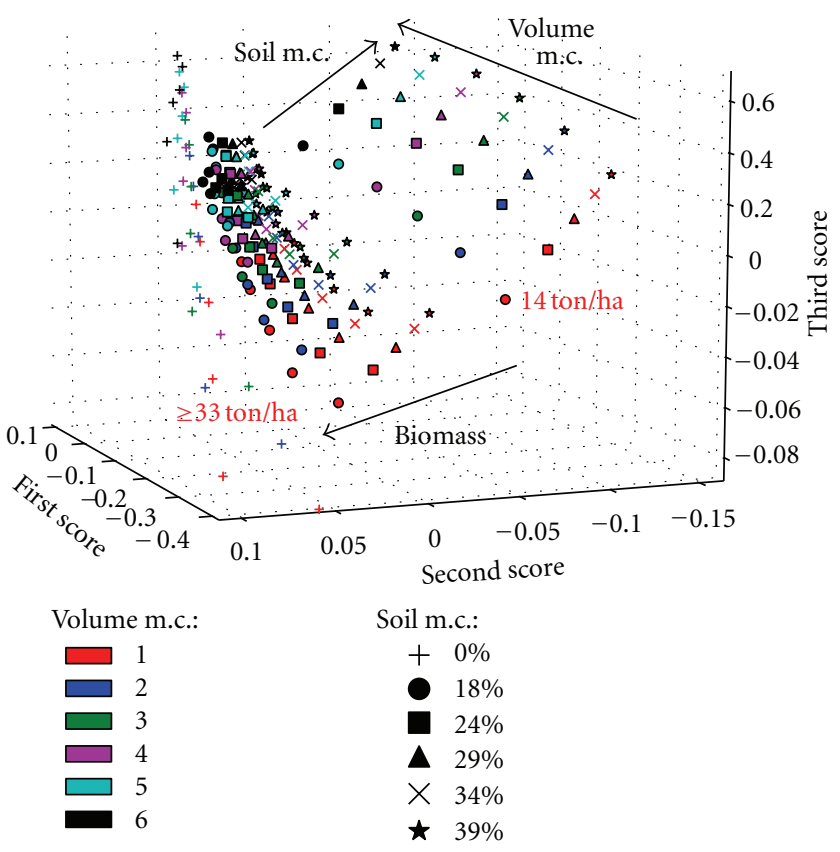

Figure 10: Result of a PCA on radar HH, HV, and VV radiometry. In the $3 \mathrm{D}$ representation of the 3 third vectors, the color corresponds to the volume m.c. and the symbol to the soil m.c. The corresponding biomass from 14 to 133 ton/ha is indicated in the figure.

\section{Radar and Optical Complementarity with Unsupervised Data Analysis}

With the radar and the optical outputs of the previous simulations on two types of forest, analyses of radar and optical data have been done with an unsupervised data analysis: the Principal Component Analysis (PCA).

All combinations of biophysical parameters were taken into account for a total of 216 simulations: 6 biomasses, 6 soil moisture content, and 6 leaves and branches moisture content, as previously indicated in Table 1.

The PCA principle [20] is to represent the data in a new base which maximize the variance along the axes. The axes are given in a decreasing order of variance: the first one is the axis that maximizes the variance; the second one is the axis perpendicular to the first one that maximizes the variance, and so on for the other axes. These three first axes only will be plotted, in order to link them to biophysical parameters.

5.1. Forest without Leaves. In a first time, the scene with soil, trunks, and branches only will be considered. This situation is an approximation of a winter temperate forest but without snow in the scene.

We can see in Figure 10 that with the polarimetric radar radiometry only as an input of the PCA, there is a separation for soil moisture content, for volume moisture content, and for biomasses lower than 50 ton/ha. It seems that there is poor sensitivity for high biomasses. 


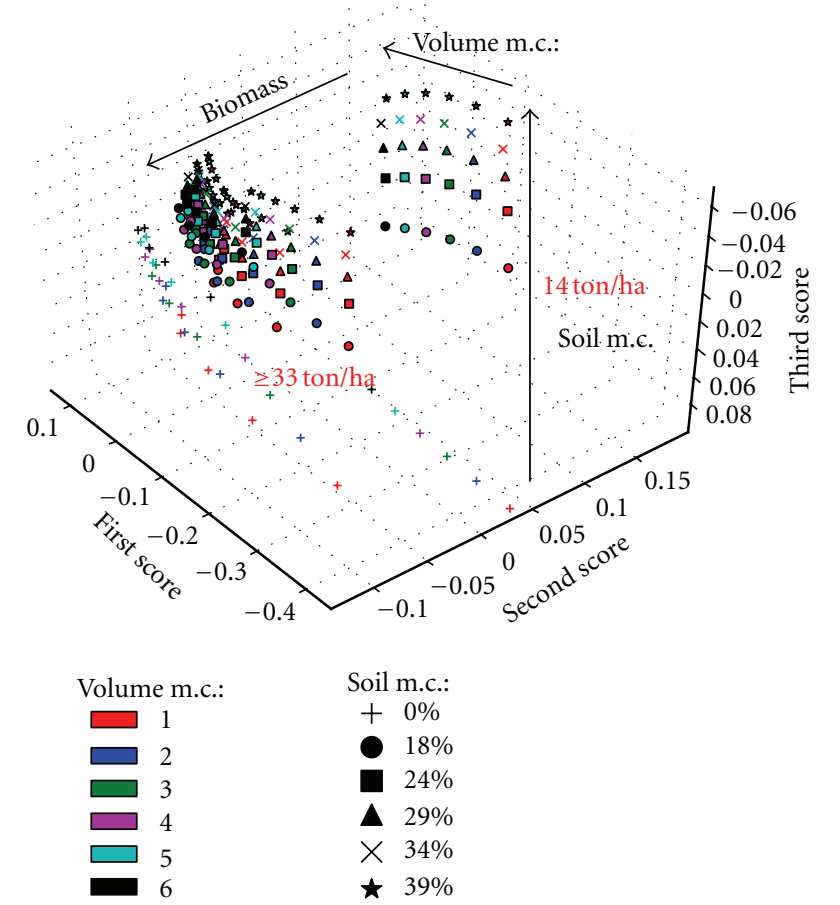

FIgURE 11: Result of a PCA on radar $\mathrm{HH}, \mathrm{HV}$, VV radiometry and optical NINSOL index for forest without leaves.

For Figure 11, the optical index NINSOL is added to the input list of the data analysis. NDVI and GVMI are not computed in this case because there is no leaf in the scene.

We can notice that the biomass separation and the volume moisture content separation are similar, while the soil moisture separation is improved for low biomasses. This result was expected because the NINSOL has been designed to be sensitive to soil moisture content and so brings information on this parameter variation in the data analysis.

However, it seems that this information was already included in the radar results, that is, the fact that $\mathrm{HH}$ backscattering is sensitive to soil moisture content.

5.2. Forest with Needles. Now, simulated data on forest with needles are injected as input of the PCA. Radar data are simulated at P-band, with a wavelength of roughly $70 \mathrm{~cm}$. Consequently, for radar simulations, the presence of leaves has no effect on the results, and then the results with or without leaves are the same.

With optical indexes only, as in Figure 12, there is a very good separation for volume moisture content and a good separation for soil moisture content lower than $29 \%$.

This result may be explained by the fact that NDVI and GVMI are, respectively, sensitive to LAI, and so biomass, and to leaves moisture content. But the leaves are occulting the soil, and so the NINSOL which has been designed for bare soils cannot be sensitive to soil moisture content, as it was for forest without leaves.

In Figure 13, radar data are incorporated in the PCA. In this case, we can notice that the separation is in the same
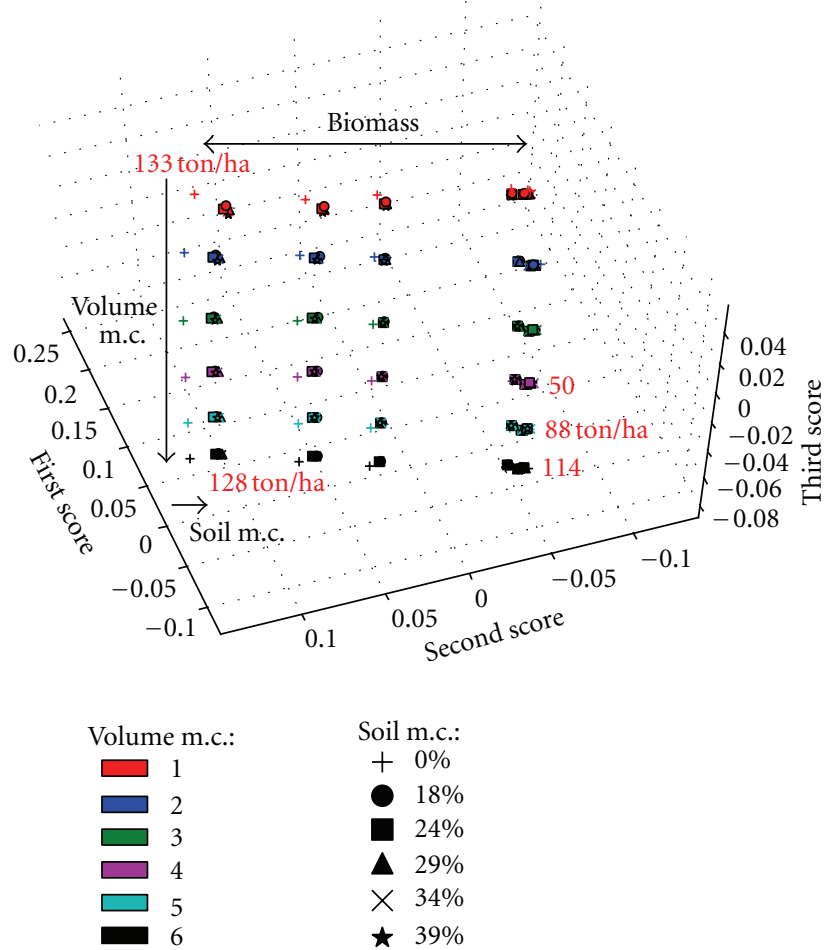

FIGURe 12: Result of a PCA on optical NDVI, GVMI, and NINSOL indexes for forests with needles.

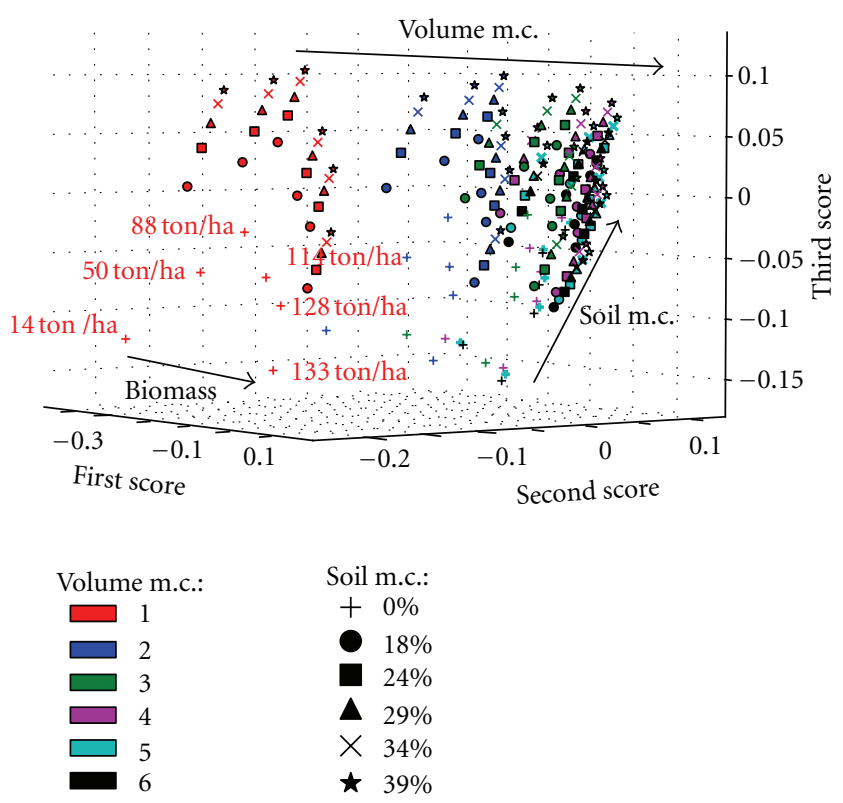

Figure 13: Result of a PCA on radar HH, HV, VV radiometry and optical NDVI, GVMI, and NINSOL indexes, for forests with needles.

order for biomass and volume moisture content as with optical data only as input as the PCA.

In addition, the soil moisture content is well separated in this case. This shows the interest of the fusion of radar and optical data to retrieve biophysical parameters. 


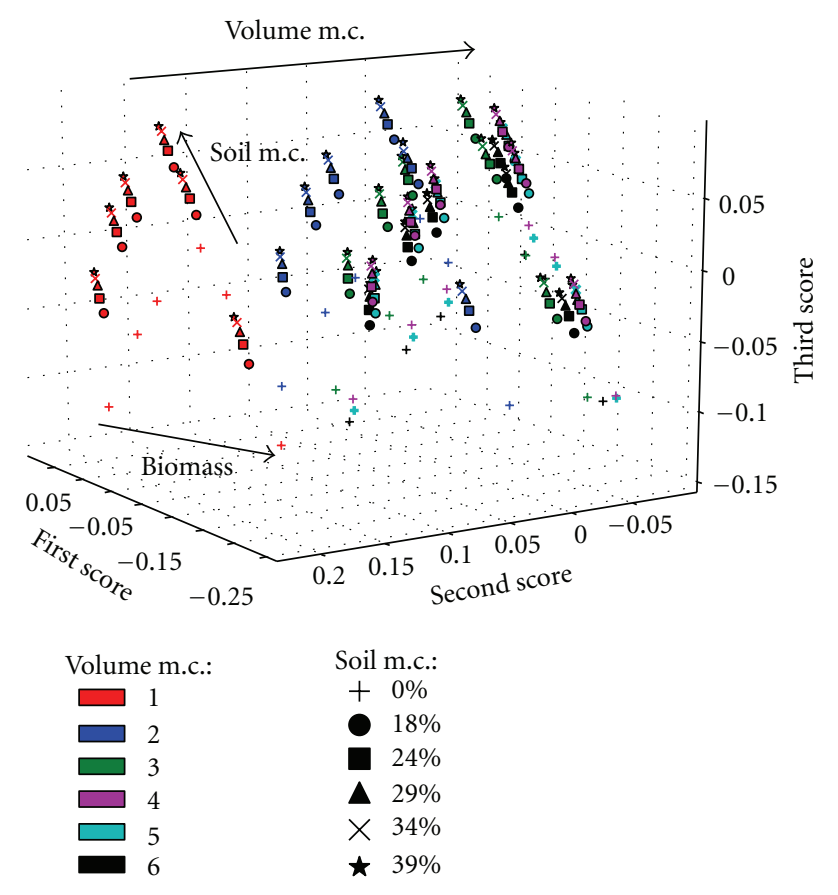

Figure 14: Result of a PCA on radar $\mathrm{HH}, \mathrm{HV}$ radiometry and optical NDVI, GVMI, and NINSOL indexes, for forests with needles. The groups of same biomass are the same as in Figure 13.

Finally, a PCA with the same inputs as previously but without VV was performed, and its result is displayed in Figure 14.

One can see that the separation is the same as when VV is one of the inputs, with close results for soil moisture content and volume moisture content. Only the biomass is a little less well separated. This result is very interesting for evaluating the performances while working with dual-polarization or compact-polarization radar [21].

We have seen in this section that the unsupervised data analysis was well separating the data along the three biophysical parameters. This result allows us to see how the same data will evolve with a supervised data analysis.

\section{Radar and Optical Complementarity with Supervised Data Analysis}

The considered data analysis is the Canonical Analysis [22]. The principle of this analysis is close to the PCA but with the objective of minimizing the variance of data inside some classes and maximizing the variance between the classes. Here again, the data are represented in a new base in which the axes are given in a decreasing order of variance.

The three radar intensities and three optical indexes previously computed for forests with needles are the input of the supervised data analysis.

The first Canonical Analysis was performed with the three radar inputs and with the three optical inputs previously computed on forest with needles, and classes of constant biomass were done.

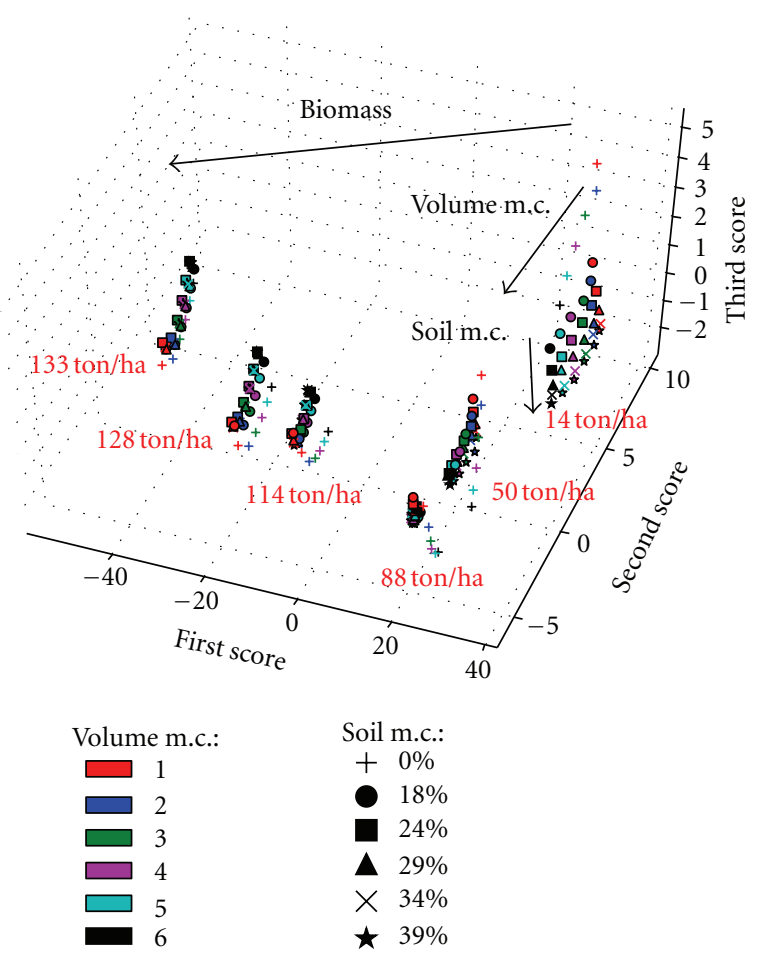

Figure 15: Result of a Canonical Analysis performed on biomass, with radar $\mathrm{HH}, \mathrm{HV}, \mathrm{VV}$ radiometry and optical NDVI, GVMI and NINSOL indexes.

In Figure 15, the separation along the biomass parameter is obvious for the first score. In addition, we can see a good separation of soil moisture content and volume moisture content for very low biomass. This can be explained by the fact that after having found the first axis that will maximize the separation of data along the biomass parameter, the canonical analysis will act like a PCA and will find the effects of the two other parameters in the data.

In order to separate the data along the three parameters, two other Canonical Analyses were performed with classes of same soil moisture content for the second Canonical Analysis and classes of same volume moisture content for the third one.

A data representation is proposed with the first score of the three Canonical Analyses displayed along the three axes.

In Figure 16, the very good separation of the three parameters can be seen. The separation is almost performed along orthogonal planes, which will facilitate future parameter retrieval. The only disadvantage is the need for training data covering the range of the three parameters.

\section{Conclusion}

A parallel model for simulating electromagnetic scattering and optical reflectance spectrum of forests has been presented. It starts from a growth model, and the subsequent geometrical discrete description is the common input for both optical and radar models. A sensitivity analysis has been carried out for the first time on P-band polarimetric radar scattering and on optical data separately, for forests without 


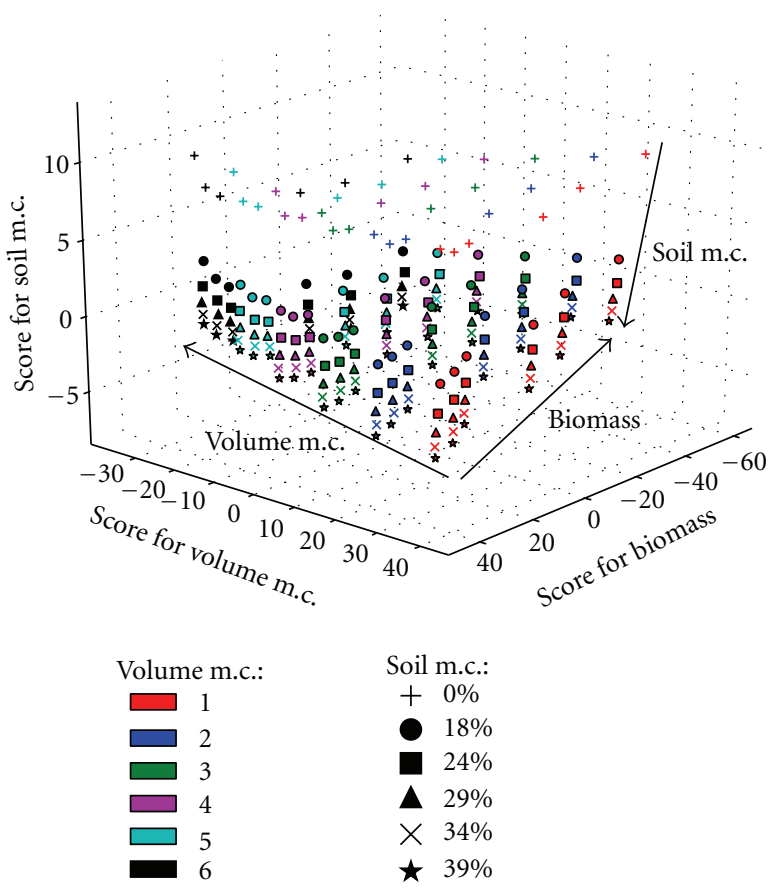

Figure 16: Result of a Canonical Analysis performed on biomass, with radar $\mathrm{HH}, \mathrm{HV}, \mathrm{VV}$ radiometry and optical NDVI, GVMI, and NINSOL indexes.

leaves and for forests with needles. Then, the sensitivity study has continued with fusion of radar and optical data with two data analyses: a nonsupervised analysis, the Principal Component Analysos, and a supervised one, the Canonical Analysis.

In this paper, it was shown that radar results, which are identical whatever the presence of leaves or needles, lead to a good separation of volume moisture content and of soil moisture content for low biomass, that is, lower than roughly 50 ton/ha. In addition, low biomasses are well separated from larger ones. On the other hand, optical data, in the case of forest with needles, leads to a good separation of the biomass and the volume moisture content, but the soil moisture content is not well separated. In the case of trees without leaves, that is, deciduous trees in winter, fusion improves slightly the radar results. In the case of trees with needles, the fusion of both radar and optical data allows a good separation of the three biophysical parameters, making possible inversion strategies based on such mixed data.

It was also shown that with such a fusion, compact polarimetry yields performances close to full polarimetry ones.

To sum up, this sensitivity analysis has exhibited the benefit which may be withdrawn from the use of parallel radar and optical models and the improvement of the inversion of forests biophysical parameters thanks to the fusion of radar and optical data.

\section{References}

[1] T. Le Toan, S. Quegan, M. W. J. Davidson et al., "The BIOMASS mission: mapping global forest biomass to better understand the terrestrial carbon cycle," Remote Sensing of Environment, vol. 115, pp. 2850-2860, 2011.

[2] R. N. Treuhaft, G. P. Asner, B. E. Law, and S. Van Tuyl, "Forest leaf area density profiles from the quantitative fusion of radar and hyperspectral data," Journal of Geophysical Research D, vol. 107, no. 21, p. 4568, 2002.

[3] P. E. Ardanuy, D. Han, and V. V. Salomonson, "The moderate resolution imaging spectrometer (MODIS) science and data system requirements," IEEE Transactions on Geoscience and Remote Sensing, vol. 29, no. 1, pp. 75-88, 1991.

[4] D. G. Goodenough, H. Chen, A. Dyk, G. Hobart, and A. Richardson, "Fusion of radar and hyperspectral data for forest applications," in Proceedings of the 59th International Astronautical Congress (IAC '08), pp. 2895-2899, Glasgow, Scotland, October 2008.

[5] C. Albinet, P. Borderies, and S. Fabre, "Radar and optical parallel modelling of forest remote sensing data," International Journal of Remote Sensing Applications, vol. 2, no. 4, 2012.

[6] K. Saleh, A. Porté, D. Guyon, P. Ferrazzoli, and J. P. Wigneron, "A forest geometric description of a maritime pine forest suitable for discrete microwave models," IEEE Transactions on Geoscience and Remote Sensing, vol. 43, no. 9, pp. 2024-2034, 2005.

[7] P. Borderies, L. Villard, C. Albinet, and N. Floury, "Forest modelling At P-band: temperate pine forest," in Proceedings of the International Geoscience and Remote Sensing Symposium, Munich, Germany, 2012.

[8] L. Villard, I. Hajnsek, and K. Papathanassiou, "Pol-InSAR simulations in forest bistatic scattering," in Proceedings of the 7th European Conference on Synthetic Aperture Radar (EUSAR '08), 2008.

[9] J. P. Gastellu-Etchegorry, E. Martin, and F. Gascon, "DART: a 3D model for simulating satellite images and studying surface radiation budget," International Journal of Remote Sensing, vol. 25, no. 1, pp. 73-96, 2004.

[10] ASTER Spectral Library-Version 2.0, http://speclib.jpl.nasa .gov/.

[11] S. Jacquemoud and F. Baret, "PROSPECT: a model of leaf optical properties spectra," Remote Sensing of Environment, vol. 34, no. 2, pp. 75-91, 1990.

[12] M. Lang, A. Kuusk, T. Nilson, T. Lukk, M. Pehk, and G. Alm, "Reflectance spectra of ground vegetation in sub-boreal forests," Tartu Observatory, Estonia, 2002, http://www.aai.ee/ bgf/ger2600/.

[13] A. Lesaignoux, Estimation de l'Humidité de Surface des Sols Nus à Partir de l'Imagerie Hyperspectrale sur le Domaine Optique 0.4-14 $\mu m$ [Ph.D. dissertation], Institut Supérieur de l'Aéronautique et de l'Espace, 2010.

[14] T. Loke, "High resolution, high speed hyperspectral cameras for laboratory industrial and airborne applications," Norsk Elektro Optikk.

[15] J. Way, J. Paris, M. C. Dobson et al., "Diurnal change in trees as observed by optical and microwave sensors: The EOS Synergism Study," IEEE Transactions on Geoscience and Remote Sensing, vol. 29, no. 6, pp. 807-821, 1991.

[16] F. J. Kriegler, W. A. Malila, R. F. Nalepka, and W. Richardson, "Preprocessing transformations and their effects on multispectral recognition," in Proceedings of the 6th International Symposium on Remote Sensing of Environment, pp. 97-131, 1969.

[17] P. Ceccato, N. Gobron, S. Flasse, B. Pinty, and S. Tarantola, "Designing a spectral index to estimate vegetation water content from remote sensing data: Part 2. Validation and applications," Remote Sensing of Environment, vol. 82, no. 23, pp. 198-207, 2002. 
[18] A. Lesaignoux, S. Fabre, X. Briottet, and A. Olioso, "Estimation of Soil Moisture Content of bare soils from their spectral optical properties in the $0.4-12 \mu \mathrm{M}$ spectral domain," in Proceedings of the 30th IEEE International Geoscience and Remote Sensing Symposium (IGARSS '10), pp. 3861-3864, July 2010.

[19] D. M. Gates, "Water relations of forest trees," IEEE Transactions on Geoscience and Remote Sensing, vol. 29, no. 6, pp. 836$842,1991$.

[20] K. Pearson, "On lines and planes of closest fit to systems of points in space," Philosophical Magazine, vol. 2, no. 6, pp. 559$572,1901$.

[21] M. L. Truong-Loï, P. Dubois-Fernandez, E. Pottier, A. Freeman, and J. C. Souyris, "Potentials of a compact polarimetric SAR system," in Proceedings of the 30th IEEE International Geoscience and Remote Sensing Symposium (IGARSS '10), pp. 742-745, Honolulu, Hawaii, USA, July 2010.

[22] C. Chatfield and A. J. Collins, Introduction to Multivariate Analysis, Chapman and Hall, 1980. 

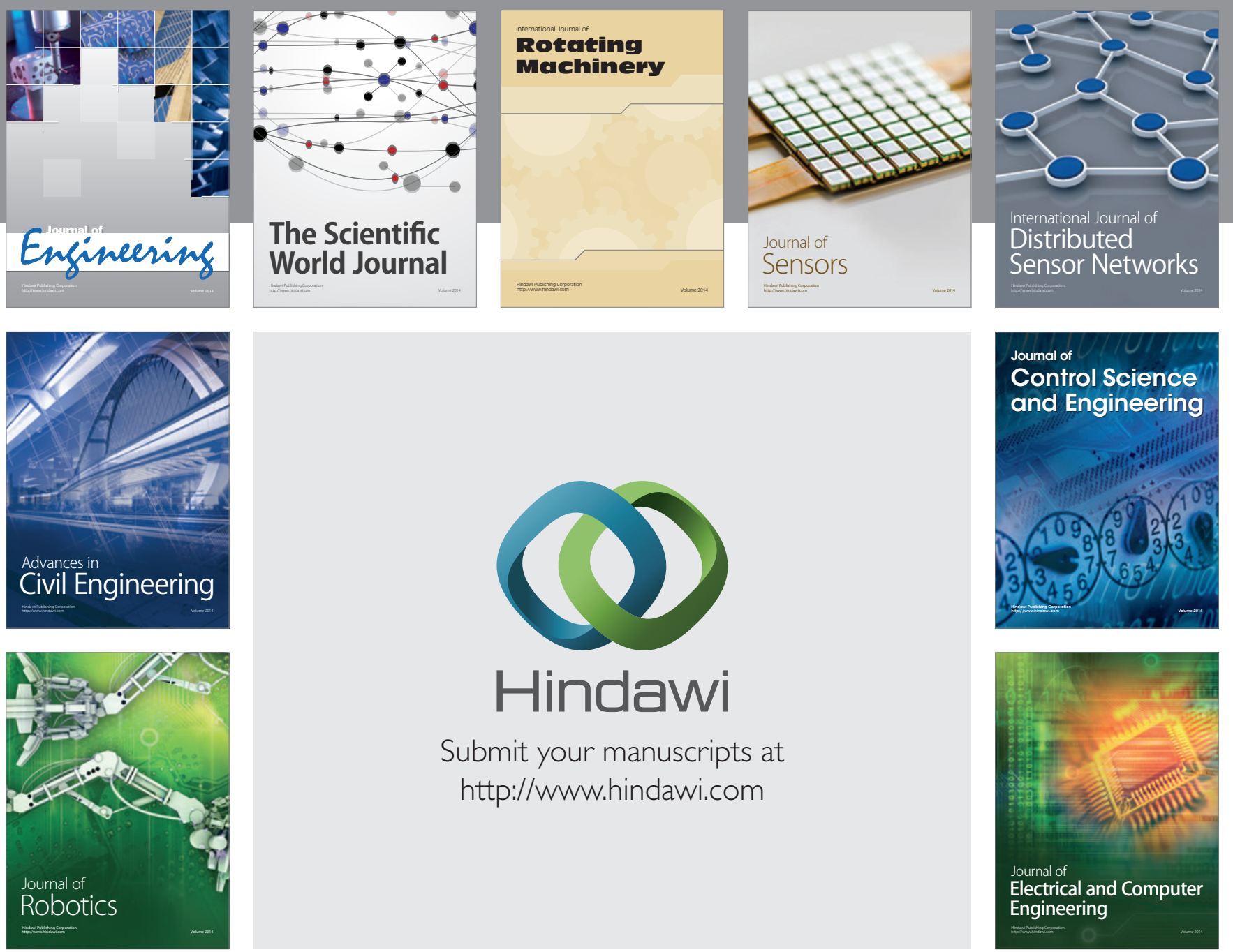

Submit your manuscripts at

http://www.hindawi.com
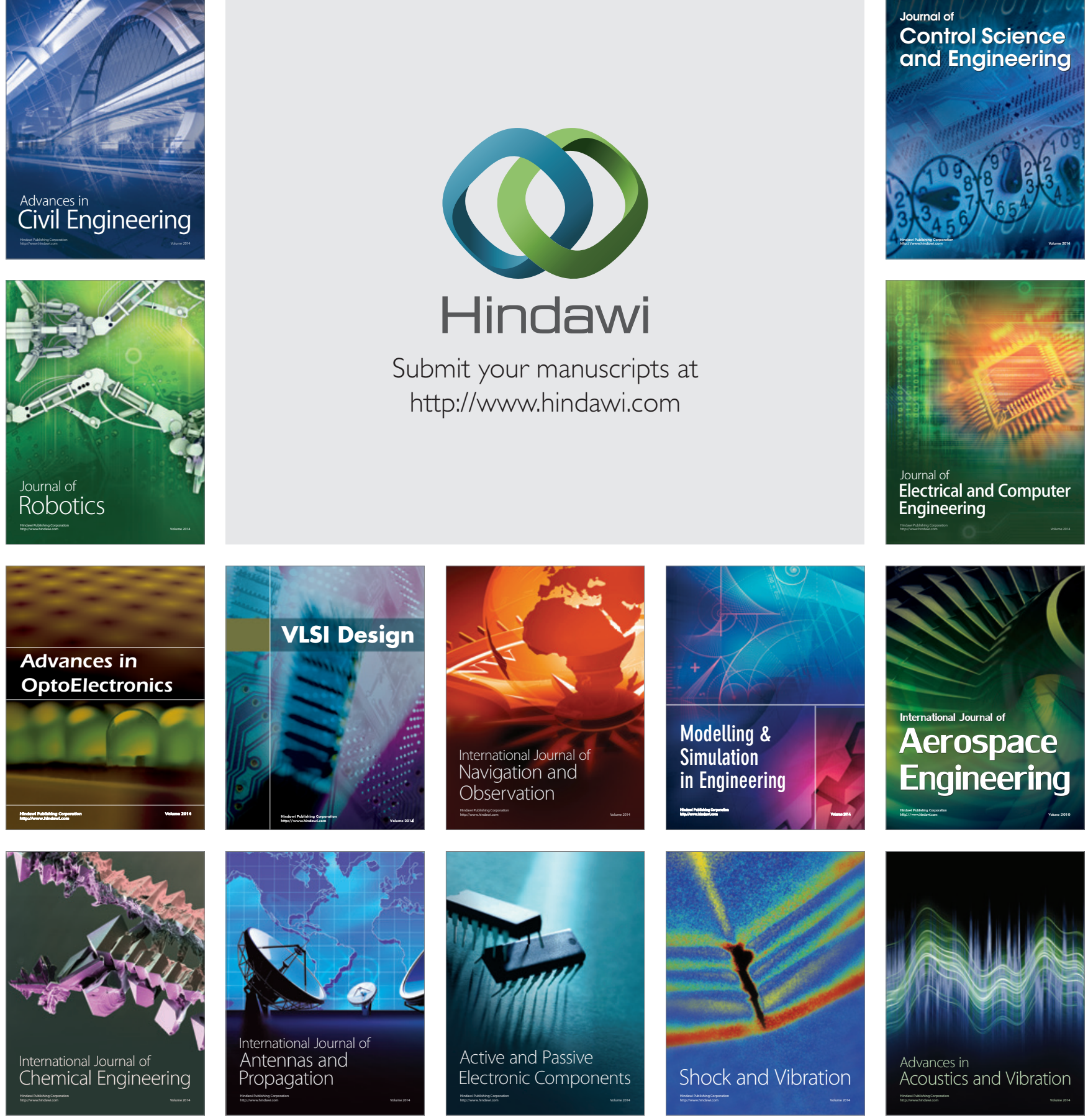